\title{
Immune (Idiopathic) Thrombocytopenic Purpura Diagnosed in Pregnancy: A Case Report and Review of Management
}

\author{
Eugene Youjin Huang ${ }^{\mathrm{a}, \mathrm{c}}$, Lay Kok Tan ${ }^{\mathrm{b}}$
}

\begin{abstract}
Immune (idiopathic) thrombocytopenic purpura (ITP) is an uncommon, but important cause of thrombocytopenia in pregnancy. It is a diagnosis of exclusion, and management should be based on a multidisciplinary care approach. ITP is characterized by moderate-to-severe thrombocytopenia commonly diagnosed in the first or early second trimester of pregnancy. The severity of thrombocytopenia has adverse implications on both maternal and fetal well-being. This paper is based on a case seen and managed in our institution and aims to discuss the various causes of thrombocytopenia and its implications in pregnancy as well as management of ITP in pregnancy based on current evidence and guidelines.
\end{abstract}

Keywords: Immune thrombocytopenic purpura; Idiopathic thrombocytopenic purpura; ITP; Pregnancy; Management

\section{Introduction}

We report a case of ITP diagnosed in pregnancy. The patient was a 29 -year-old primigravida who was transferred at 21 weeks gestation from a private healthcare institution for thrombocytopenia detected on routine clinical testing. Collaboration between the obstetrician and hematologist in our case was important to provide a smooth antenatal journey to ensure good maternal and fetal outcomes.

\section{Case Report}

Our patient had no past medical history, and her antenatal

\footnotetext{
Manuscript accepted for publication June 30, 2015

aDivision of Obstetrics and Gynecology, KK Women's and Children's Hospital, Singapore

bepartment of Obstetrics and Gynecology, Singapore General Hospital, Singapore

${ }^{\mathrm{c} C}$ Corresponding Author: Eugene Youjin Huang, Division of Obstetrics and Gynecology, KK Women's and Children's Hospital, 100 Bukit Timah Road, 229899, Singapore. Email: huangeugene@gmail.com
}

doi: http://dx.doi.org/10.14740/jmc2205w follow-up was uneventful prior to the transfer. However, she complained of intermittent and worsening gum bleeding for a few months into her pregnancy. A platelet count was performed, and the value was $12 \times 10^{9} / \mathrm{L}$. Peripheral blood film showed marked thrombocytopenia, but other blood cell types were normal. Direct Coombs' test was negative for hemolysis, and lactate dehydrogenase (LDH) level was normal. Her blood pressure was normal and there was no evidence of proteinuria. Her baseline liver function, renal function, thyroid function, coagulation screening and uric acid levels were within normal limits. Screening tests for infectious diseases such as hepatitis B, hepatitis C, HIV, EBV, CMV and dengue were all negative. Autoimmune markers such as antinuclear antibody, anti-dsDNA antibody and anticardiolipin antibodies were also negative.

In view of the acute presentation and with no other obvious causes of thrombocytopenia, a working diagnosis of ITP was made, and she was started on intravenous (IV) immunoglobulin and oral prednisolone. Her platelet counts rose to 36 $\times 10^{9} / \mathrm{L}$ before discharge from hospital. She was subsequently followed up in our obstetric high risk and hematology clinics.

Throughout the antenatal period, she had multiple hospital admissions due to low platelet counts and bleeding, e.g., epistaxis and gum bleeding. Infusions of IV immunoglobulin and adjustment of her steroid doses had achieved an acceptable platelet count during these admissions. At 29 weeks gestation, she presented with painless antepartum hemorrhage with no cervical dilatation or uterine contractions. Antenatal corticosteroids were given in view of vaginal bleeding and the risk of preterm labor. An ultrasound scan showed a normal growing fetus in a cephalic presentation with no placenta previa or retroplacental clots. Her symptoms resolved subsequently.

At 33 weeks, she was again admitted for low platelet counts. IV immunoglobulin and steroids (including pulse dexamethasone) were given. Her platelet counts were in the range of 15 $40 \times 10^{9} / \mathrm{L}$, and were fluctuating to low levels despite treatment. A repeat ultrasound scan showed a normal fetus with normal Doppler studies and an estimated fetal weight of $2.6 \mathrm{~kg}$. In view of increasing resistance to conventional ITP treatment and good fetal weight, plan for delivery was discussed at 35 weeks with the patient and her hematologist after weighing the risks and benefits of having a premature baby versus worsening thrombocytopenia that could potentially cause maternal morbidity and fetal thrombocytopenia due to transfer of antiplatelet antibodies 
Table 1. Causes of Thrombocytopenia in Pregnancy [2]

\begin{tabular}{ll}
\hline Gestational thrombocytopenia & $\begin{array}{l}\text { Antiphospholipid antibody } \\
\text { syndrome }\end{array}$ \\
\hline Preeclampsia/HELLP syndrome & $\begin{array}{l}\text { Disseminated intravascular } \\
\text { coagulation }\end{array}$ \\
Acute fatty liver of pregnancy & Viral infection \\
TTP & Nutritional deficiency \\
HUS & Drug use \\
SLE & Primary bone marrow disorder \\
\hline
\end{tabular}

transplacentally. As there was difficulty to time delivery with optimal platelet levels, induction of labor was deemed unsuitable and decision was made to deliver the baby by elective cesarean section at $35+2$ weeks with platelet and NovoSeven ${ }^{\circledR}$ cover. Her platelet count was $31 \times 10^{9} / \mathrm{L}$ preoperatively. Risk of postpartum hemorrhage and possible need for medical treatment, uterine compression sutures, radiological intervention and cesarean hysterectomy were discussed with the patient.

The cesarean section was complicated by uterine atony, and various oxytocics and a B-Lynch suture were needed to contract the uterus. She was otherwise well postoperatively and was discharged well 7 days after surgery. Her platelet count on discharge was $98 \times 10^{9} / \mathrm{L}$.

\section{Discussion}

Thrombocytopenia is common and occurs in about $10 \%$ of all pregnancies [1]. There are various causes of thrombocytopenia in pregnancy, and while some are not associated with adverse pregnancy outcomes, some are associated with significant maternal/fetal morbidity and mortality. As ITP is a diagnosis of exclusion, it is therefore prudent to consider all causes of thrombocytopenia before making a diagnosis of ITP, as management is different depending on the cause. The causes of thrombocytopenia are listed in Table 1 [2].

ITP occurs in $0.1-0.2 \%$ of all pregnancies and is responsible for $5 \%$ of all cases of thrombocytopenia diagnosed in pregnancy [3]. We will go through a brief overview of the common causes of thrombocytopenia before dwelling on the management of ITP in pregnancy.

Gestational thrombocytopenia is the most common cause, and it accounts for $65-80 \%$ of cases [4]. Due to physiological changes in pregnancy, such as increased blood volume, platelet activation and clearance [5], mild thrombocytopenia occurs during late pregnancy, especially during the third trimester. Most importantly, patients remain asymptomatic, and the platelet counts are usually more than $70 \times 10^{9} / \mathrm{L}$, with about two-thirds being $130-150 \times 10^{9} / \mathrm{L}$ [6]. Gestational thrombocytopenia is not associated with fetal thrombocytopenia, and it spontaneously resolves after delivery.

Preeclampsia has to be considered in a patient with thrombocytopenia in the third trimester associated with raised blood pressure $(\geq 140 / 90 \mathrm{~mm} \mathrm{Hg})$ and significant proteinuria $>0.3 \mathrm{~g} /$ day. The triad of microangiopathic hemolytic anemia, abnor- mal liver function (AST $\geq 70 \mathrm{IU} / \mathrm{L}$ ), and thrombocytopenia with a platelet count less than $100 \times 10^{9} / \mathrm{L}$ constitutes the diagnosis of hemolysis, elevated liver enzymes and low platelets (HELLP) syndrome [7]. Other markers include an elevated $\mathrm{LDH} \geq 600 \mathrm{IU} / \mathrm{L}$ and raised bilirubin levels $\geq 17.1 \mu \mathrm{mol} / \mathrm{L}[8]$. Both conditions require medical stabilization followed by delivery.

Thrombotic thrombocytopenic purpura (TTP) and hemolytic uremic syndrome (HUS) are a continuum, and both are manifestations of a similar mechanism of microvascular platelet aggregation. Although rare, these two conditions should be considered in pregnant women with thrombocytopenia. TTP is characterized by a pentad of microangiopathic hemolytic anemia, thrombocytopenia, neurological abnormalities, fever and renal dysfunction, and can occur in any trimester [9]. HUS is predominated by renal abnormalities rather than neurological abnormalities and occurs most commonly in the postpartum period $(>90 \%)$ [10]. The manifestations of TTP and HUS may be confused with preeclampsia or HELLP syndrome, but hypertension is not common in TTP and HUS, and there is no coagulopathy.

Autoimmune conditions such as systemic lupus erythematosus (SLE) and antiphospholipid antibody syndrome may first appear or increase in severity during pregnancy and thrombocytopenia occurs in $10-30 \%$ of cases. It is important to note that about $25 \%$ of SLE patients have antiphospholipid antibodies which are associated with fetal loss and the need for anticoagulation [8]. Positive screening for autoimmune antibodies in association with condition-specific signs and symptoms help define the definite cause. Joint management with a rheumatologist is prudent.

Other causes of thrombocytopenia in pregnancy include: 1) disseminated intravascular coagulation, especially in the context of placental abruption, massive hemorrhage secondary to uterine rupture, retention of a intrauterine stillbirth and amniotic fluid embolism; 2) acute fatty liver of pregnancy, with typical presentations of malaise, anorexia, nausea, vomiting, epigastric or right upper quadrant pain, mental state changes and cholestasis; 3) viral infections such as HIV and hepatitis C-induced thrombocytopenia; 4) drug causes, such as heparininduced thrombocytopenia or use of recreational drugs such as cocaine which has been associated with a syndrome resembling HELLP, and may be accompanied by a transient development of profound thrombocytoenia [11]; and 5) primary bone marrow disorder, usually associated with other blood cell dyscrasias. A bone marrow examination will be necessary to make a diagnosis of the type of bone marrow disorder.

In our case, all the common causes had been excluded by performing initial blood investigations and in the context of the history, physical findings and early presentation, a diagnosis of ITP was made and appropriate treatment rendered. Generally speaking, thrombocytopenia that occurs in the first or early second trimester or in cases with moderate-to-severe low platelet counts, the patient should be investigated for secondary causes. ITP can only be diagnosed if all the above-mentioned causes are excluded.

The management of ITP in pregnancy requires close collaboration between the obstetrician, hematologist, anesthetist and neonatologist. Upon diagnosis, the severity of thrombo- 
cytopenia should be ascertained, and platelet counts should be increased and stabilized to a safe level in pregnancy, especially during delivery and provision of epidural anesthesia. Patients will require close monitoring, with routine blood pressure and weight measurements, urine dipstick for protein and serial platelet counts at every visit [8]. Treatment should be instituted, if platelets fall to an unsafe low level or if the patient is symptomatic for bleeding. The American Society of Hematology (ASH) and the British Committee for Standards in Hematology (BCSH) - General Hematology Task Force guidelines provide guidance to what is considered a safe platelet level for delivery and procedures, as well as when to institute treatment. The ASH suggests a safe platelet count of at least $50 \times 10^{9} / \mathrm{L}$ for both vaginal delivery and cesarean section. Platelets less than $10 \times 10^{9} / \mathrm{L}$ or platelets $10-30 \times 10^{9} / \mathrm{L}$ in the second/third trimester or symptomatic bleeding are indications for treatment [12]. The BCSH suggests a safe platelet count of at least $50 \times 10^{9} / \mathrm{L}$ and $80 \times 10^{9} / \mathrm{L}$ for vaginal delivery and cesarean section respectively. A minimum platelet count of $80 \times 10^{9} / \mathrm{L}$ is considered safe for epidural analgesia. Platelets less than $20 \times$ $10^{9} / \mathrm{L}$ in any trimester is an indication for treatment under the $\mathrm{BCSH}$ guidelines [13].

The mainstay medical treatment for ITP in pregnancy does not differ from non-pregnant patients, except for the use of controversial drugs such as immunomodulating agents, monoclonal antibodies and thrombopoietic agents, which are not well studied and do not have well-established safety data in pregnancy. The standard treatment for ITP is corticosteroids, with a starting dose of $1 \mathrm{mg} / \mathrm{kg} /$ day (weight based on pre-pregnancy weight), after which the dose should be titrated to the lowest effective dose to achieve remission [8]. If rapid rise of the platelet count is necessary, then IV immunoglobulin would be the treatment of choice. IV immunoglobulin is less likely to cause adverse side effects associated with corticosteroids like diabetes, hypertension, excessive weight gain and osteoporosis. However, the use of IV immunoglobulin is limited by its high cost and transient response. For patients who do not respond to single therapy, a combination of corticosteroids and IV immunoglobulin may be considered. IV steroids such as pulse dexamethasone and methylprednisolone may be used in lieu of oral steroids in such cases [14].

The use of other medical treatments has also been tested. These treatments are still controversial due to lack of safety data and true efficacy. If these agents are to be used, it has been recommended not to use them in the first trimester when organogenesis occurs. Examples of such treatments include as follows. 1) IV anti-D has been used in women in their second and third trimesters. One study reported that six out of eight women had successful responses, with no maternal or fetal effects and no evidence of fetal hydrops [15]. 2) Immunomodulating drugs such as azathioprine have been shown to work and found to be safe in pregnant women with renal transplants [16]. Cytotoxic drugs such as cyclophosphamide cannot be used due to its teratogenic potential. 3) Monoclonal antibodies such as rituximab have been used to treat B-cell lymphoma and recently found to be able to treat ITP. It has been found not to be associated with fetal malformations, although there are a few case reports which documented infants with abnormal B-cell development in the first year of life [17]. 4) Use of thrombopoietic agents such as eltrombopag or romiplostim have been studied, but there is still little experience in their use in pregnancy [2]. For cases refractory to conventional treatment or if treatment toxicities are unacceptable, splenectomy can be performed in the second trimester when fetal and anesthetic risks are minimal [18]. The BCSH has recommended the laparoscopic approach for splenectomy.

With regards to peripartum management in patients with ITP, the risk of maternal hemorrhage is minimized by ensuring minimum platelet counts required for vaginal delivery, cesarean section and epidural analgesia as stipulated by the ASH or BCSH guidelines are met $[12,13]$. The transplacental passage of maternal antiplatelet antibodies in pregnancy can cause fetal thrombocytopenia, and the most feared consequence of this is fetal intracranial hemorrhage during vaginal delivery. The risk of fetal thrombocytopenia has been reviewed in a metaanalysis by Burrows et al, which showed that $10.1 \%$ of infants born to mothers with ITP had platelet counts below $50 \times 10^{9} / \mathrm{L}$ and $4.2 \%$ below $20 \times 10^{9} / \mathrm{L}$ [19]. Fujimura et al noted that the risk of intracranial hemorrhage in the offspring of patients with ITP is very low $(<1 \%)[20]$. Various studies have also shown no correlation between maternal ITP status, platelet counts and the development of intracranial hemorrhage. There is no role of fetal blood sampling or cordocentesis to predict fetal thrombocytopenia [8]. Evidence so far has suggested that the most reliable predictor of fetal thrombocytopenia is a prior history of thrombocytopenia in an older sibling at delivery [21].

It was previously believed that cesarean section reduced the risk of fetal intracranial hemorrhage associated with trauma during vaginal birth. Cook et al reappraised the peripartum management of patients with ITP and found no association between the mode of delivery and the risk of intracranial hemorrhage [22]. As evidence has suggested poor correlation between maternal ITP and development of intracranial hemorrhage, it is recommended that cesarean section be performed for obstetric indications only.

The newborn should be assessed for thrombocytopenia with serial platelet counts for 1 week postpartum, as well as assess for intracranial hemorrhage. Brain imaging should be performed if neonatal platelet count is below $50 \times 10^{9} / \mathrm{L}$. It has been shown that neonatal platelet counts fall to a nadir by day 2 . By day 7 , the platelet counts should have begun to rise or stabilized [23]. ASH has recommended that infants with platelet counts below $20 \times 10^{9} / \mathrm{L}$ or symptomatic for bleeding receive IV immunoglobulin [12]. The use of steroids is controversial due to predisposition to neonatal sepsis [24].

\section{Conclusion}

Thrombocytopenia complicates $10 \%$ of all pregnancies [1], and fortunately, the majority of cases are benign and gestationrelated. There are, however, other causes which can potentially increase both maternal and fetal morbidity and mortality. When thrombocytopenia is diagnosed, a systematic approach is required to ascertain the cause. Gestational thrombocytopenia or ITP can only be diagnosed if other causes are excluded. Gestational thrombocytopenia is mild and does not have adverse effects on the mother or fetus, does not require treatment 
and usually resolves after delivery. On the other hand, ITP is associated with moderate-to-severe thrombocytopenia which increases the risks of maternal hemorrhage as well as fetal thrombocytopenia and potentially, fetal intracranial hemorrhage. Treatment is needed in ITP and is no different from nonpregnant individuals, except for non-conventional drugs such as cytotoxics or thrombopoietic agents. Peripartum management involves optimizing platelet counts for delivery and procedures such as epidural analgesia. Well-controlled ITP with adequate platelet counts is not a contraindication to vaginal delivery, and cesarean section should only be performed based on obstetric indications only. There is also no strong association between maternal ITP and the development of intracranial hemorrhage in the newborn, making predictability of hemorrhage difficult. It is thus recommended to monitor the infant with serial platelet counts and assessing for intracranial bleeding postpartum. Treatment should be instituted if the platelet counts are low, or if the infant is symptomatic.

\section{Grant Support}

No grant support or assistance required for this report.

\section{References}

1. McCrae K. Thrombocytopenia in pregnancy. In: Michelson A, editor. Platelets. New York: Elsevier. 2006.

2. Terry B. Gernsheimer. Thrombocytopenia in pregnancy: is this immune thrombocytopenia or ? American Society of Hematology. 2012.

3. Provan D, Newland A. Idiopathic thrombocytopenic purpura in adults. J Pediatr Hematol Oncol. 2003;25(Suppl 1):S34-38.

4. Sainio S, Kekomaki R, Riikonen S, Teramo K. Maternal thrombocytopenia at term: a population-based study. Acta Obstet Gynecol Scand. 2000;79(9):744-749.

5. Matthews JH, Benjamin S, Gill DS, Smith NA. Pregnancy-associated thrombocytopenia: definition, incidence and natural history. Acta Haematol. 1990;84(1):24-29.

6. Burrows RF, Kelton JG. Fetal thrombocytopenia and its relation to maternal thrombocytopenia. N Engl J Med. 1993;329(20):1463-1466.

7. Stone JH. HELLP syndrome: hemolysis, elevated liver enzymes, and low platelets. JAMA. 1998;280(6):559562.

8. Stavrou E, McCrae KR. Immune thrombocytopenia in pregnancy. Hematol Oncol Clin North Am. 2009;23(6):1299-1316.

9. McCrae KR, Cines DB. Thrombotic microangiopathy during pregnancy. Semin Hematol. 1997;34(2):148-158.

10. McCrae KR, Sadler JE, Cines DB. Thrombotic thrombocytopenic purpura and the hemolytic uremic syndrome. In: Hoffman R, Benz EJJ, Shattil SJ, editors. Hematol- ogy: basic principles and practice. Philadelphia: Elsevier, Churchill, Livingstone; 2005;2287.

11. Abramowicz JS, Sherer DM, Woods JR, Jr. Acute transient thrombocytopenia associated with cocaine abuse in pregnancy. Obstet Gynecol. 1991;78(3 Pt 2):499-501.

12. George JN, Woolf SH, Raskob GE, Wasser JS, Aledort LM, Ballem PJ, Blanchette VS, et al. Idiopathic thrombocytopenic purpura: a practice guideline developed by explicit methods for the American Society of Hematology. Blood. 1996;88(1):3-40.

13. Guidelines for the investigation and management of idiopathic thrombocytopenic purpura in adults, children and in pregnancy. Br J Haematol. 2003;120(4):574-596.

14. McCrae KR, Samuels P, Schreiber AD. Pregnancy-associated thrombocytopenia: pathogenesis and management. Blood. 1992;80(11):2697-2714.

15. Cines DB, Bussel JB. How I treat idiopathic thrombocytopenic purpura (ITP). Blood. 2005;106(7):2244-2251.

16. Bar J, Stahl B, Hod M, Wittenberg C, Pardo J, Merlob P. Is immunosuppression therapy in renal allograft recipients teratogenic? A single-center experience. Am J Med Genet A. 2003;116A(1):31-36.

17. Klink DT, van Elburg RM, Schreurs MW, et al. Rituximab administration in third trimester of pregnancy without consequences for the newborn. J Perinatol 2006;26(4):252-255.

18. Griffiths J, Sia W, Shapiro AM, Tataryn I, Turner AR. Laparoscopic splenectomy for the treatment of refractory immune thrombocytopenia in pregnancy. J Obstet Gynaecol Can. 2005;27(8):771-774.

19. Burrows RF, Kelton JG. Pregnancy in patients with idiopathic thrombocytopenic purpura: assessing the risks for the infant at delivery. Obstet Gynecol Surv. 1993;48(12):781-788.

20. Fujimura K, Harada Y, Fujimoto T, Kuramoto A, Ikeda Y, Akatsuka J, Dan K, et al. Nationwide study of idiopathic thrombocytopenic purpura in pregnant women and the clinical influence on neonates. Int J Hematol. 2002;75(4):426-433.

21. Christiaens GC, Nieuwenhuis HK, Bussel JB. Comparison of platelet counts in first and second newborns of mothers with immune thrombocytopenic purpura. Obstet Gynecol. 1997;90(4 Pt 1):546-552.

22. Cook RL, Miller RC, Katz VL, Cefalo RC. Immune thrombocytopenic purpura in pregnancy: a reappraisal of management. Obstet Gynecol. 1991;78(4):578-583.

23. Burrows RF, Kelton JG. Low fetal risks in pregnancies associated with idiopathic thrombocytopenic purpura. Am J Obstet Gynecol. 1990;163(4 Pt 1):1147-1150.

24. Christiaens GC, Nieuwenhuis HK, von dem Borne AE, Ouwehand WH, Helmerhorst FM, van Dalen CM, van der Tweel I. Idiopathic thrombocytopenic purpura in pregnancy: a randomized trial on the effect of antenatal low dose corticosteroids on neonatal platelet count. $\mathrm{Br} \mathrm{J}$ Obstet Gynaecol. 1990;97(10):893-898. 